\title{
Bäcklund transforms of the extreme Kerr near-horizon geometry
}

\author{
Reinhard Meinel and Andreas Kleinwächter \\ Theoretisch-Physikalisches Institut, University of Jena, \\ Max-Wien-Platz 1, 07743 Jena, Germany \\ E-mail: meinel@tpi.uni-jena.de, kleinwaechter@tpi.uni-jena.de
}

\begin{abstract}
We apply the method of Bäcklund transformations to generate a new hierarchy of exact solutions to the vacuum Einstein equations starting from the extreme Kerr near-horizon geometry. Solutions with extreme Kerr near-horizon asymptotics containing an arbitrary number of free parameters are included.
\end{abstract}

Dedicated to Gernot Neugebauer on the occasion of his 80th birthday

\section{Introduction}

The general relativistic solution describing a uniformly rotating disk of dust [1] admits an interesting parameter limit leading to the extreme Kerr metric outside the horizon ("exterior perspective"). From the "interior perspective", the same parameter limit can be performed after an appropriate parameter dependent coordinate transformation and then leads to a spacetime that still describes a rotating disk of dust embedded in a non-asymptotically flat vacuum exterior that approaches the extreme Kerr nearhorizon geometry at spatial infinity [2], see also [3]. The same phenomenon was observed numerically for "relativistic Dyson rings" [4. Moreover, it was shown that a black hole limit of rotating fluid bodies in equilibrium always leads to the extreme Kerr solution [5].

This motivates the systematic investigation of solutions which have the extreme Kerr near-horizon geometry, also called "extreme Kerr throat geometry" [6], as their 
asymptotics. As a first step, we derive here a new class of solutions by means of Bäcklund transformation $\ddagger$ [10, 11] using the extreme Kerr near-horizon geometry as the starting point ("seed solution") and show how the Bäcklund parameters have to be constrained in order to conserve the extreme Kerr near-horizon asymptotics. Of course, the solutions constructed in this way are pure vacuum solutions. Instead of material sources they possess some singularities; note that this guarantees consistency with results published in [12, 13]. However, by removing a portion of the solution that contains the singularities and replacing it with an appropriate non-vacuum solution, a meaningful, globally regular spacetime with extreme Kerr near-horizon asymptotics could be obtained.

The derived solutions might also be of interest within the context of the Kerr/CFT correspondence, see [14].

\section{Ernst potential of the extreme Kerr near-horizon geometry}

It is well known that the stationary and axially symmetric vacuum Einstein equations are equivalent to the Ernst equation [15, 16]

$$
(\Re \mathcal{E}) \nabla^{2} \mathcal{E}=(\nabla \mathcal{E})^{2},
$$

where the operator $\nabla$ has the same meaning as in Euclidean 3-space in which $r, \theta$ and $\phi$ are spherical coordinates. The complex Ernst potential $\mathcal{E}$ depends on $r$ and $\theta$ only. The spacetime line element reads

$$
\mathrm{d} s^{2}=f^{-1}\left[h\left(\mathrm{~d} r^{2}+r^{2} \mathrm{~d} \theta^{2}\right)+r^{2} \sin ^{2} \theta \mathrm{d} \phi^{2}\right]-f(\mathrm{~d} t+a \mathrm{~d} \phi)^{2}
$$

with $f=\Re \mathcal{E}$; the other metric functions $h$ and $a$ can also be obtained from $\mathcal{E}$. The Ernst potential of the extreme Kerr near-horizon geometry is given by [2]

$$
\mathcal{E}_{\mathrm{NHG}}=-\Omega^{2} r^{2} H(\theta), \quad H(\theta)=\frac{2(1+\mathrm{i} \cos \theta)^{2}}{1-\mathrm{i} \cos \theta}+\sin ^{2} \theta .
$$

The real constant $\Omega$ is the angular velocity of the horizon. Note that (3) belongs to a family of solutions discovered by Ernst [17].

\section{Bäcklund transformation}

Neugebauer's general Bäcklund formula [18] has been used to investigate huge classes of asymptotically flat solutions to the stationary and axially symmetric vacuum Einstein equations. However, it can be applied to non-asymptotically flat solutions as well. In general, for a given seed solution $\mathcal{E}_{0}$, the $(2 n$-fold) Bäcklund transform $\mathcal{E}$ reads [18, 19]

$\ddagger$ The method of Bäcklund transformations is closely related to other soliton-theoretic techniques for solving certain nonlinear partial differential equations and can be applied to the vacuum Einstein equations in the presence of two commuting Killing vectors, see [7, 8, 9]. 
Bäcklund transforms of the extreme Kerr near-horizon geometry

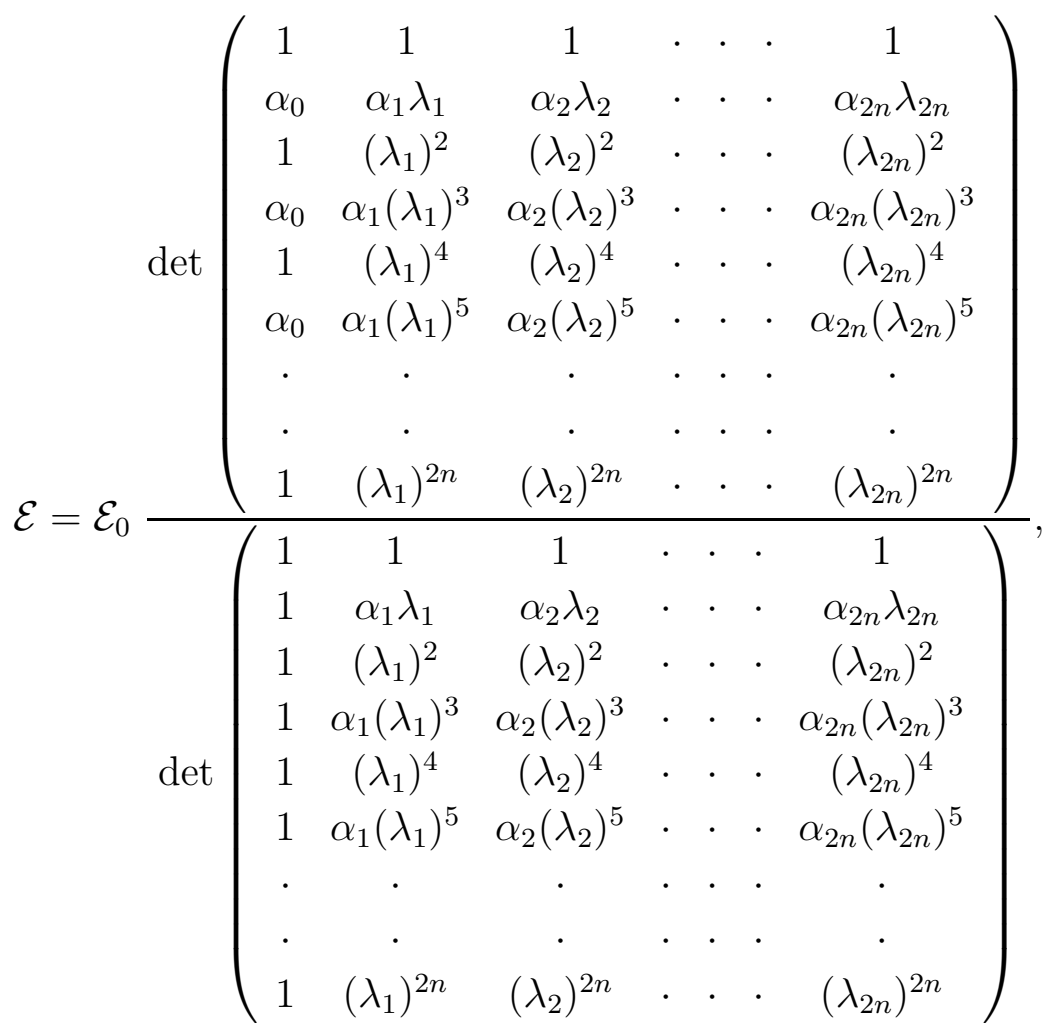

where the entries in the two $(2 n+1) \times(2 n+1)$ matrices are given by

$$
\alpha_{0}=-\frac{\mathcal{E}_{0}^{*}}{\mathcal{E}_{0}}, \quad \lambda_{i}=\sqrt{\frac{K_{i}-r \mathrm{e}^{\mathrm{i} \theta}}{K_{i}-r \mathrm{e}^{-\mathrm{i} \theta}}} \quad\left(\lambda_{i} \rightarrow \mathrm{e}^{\mathrm{i} \theta} \text { as } r \rightarrow \infty\right)
$$

and solutions $\alpha_{i}$ to the total Riccati equations

$$
\begin{aligned}
\left(\mathcal{E}_{0}+\mathcal{E}_{0}^{*}\right) \mathrm{d} \alpha_{i}= & {\left[\frac{\partial \mathcal{E}_{0}^{*}}{\partial z}\left(\alpha_{i}-\lambda_{i}\right)+\frac{\partial \mathcal{E}_{0}}{\partial z} \alpha_{i}\left(\alpha_{i} \lambda_{i}-1\right)\right] \mathrm{d} z } \\
& +\left[\frac{\partial \mathcal{E}_{0}^{*}}{\partial z^{*}}\left(\alpha_{i}-\lambda_{i}^{-1}\right)+\frac{\partial \mathcal{E}_{0}}{\partial z^{*}} \alpha_{i}\left(\alpha_{i} \lambda_{i}^{-1}-1\right)\right] \mathrm{d} z^{*}
\end{aligned}
$$

with the complex coordinates

$$
z=\mathrm{ire} \mathrm{e}^{-\mathrm{i} \theta}, \quad z^{*}=-\mathrm{i} r \mathrm{e}^{\mathrm{i} \theta} .
$$

Note that the integrability condition of (6) is equivalent to the Ernst equation for $\mathcal{E}_{0}$. Via a quotient ansatz, the Riccati equation can be reformulated as a system of linear differential equations [19]. The asymptotic fixing of $\lambda_{i}$ as given in (5) can be chosen without loss of generality since the $\lambda_{i}$ 's enter (4) only with even powers and as products $\alpha_{i} \lambda_{i}$. To any solution $\alpha_{i}$ of the Riccati equation (6) with $\lambda_{i}$ one has a corresponding solution $-\alpha_{i}$ with $-\lambda_{i}$.

The (finite) constants $K_{i}$ must either be real $\left(K_{i}=K_{i}^{*}\right)$, with the consequence $\lambda_{i}=1 / \lambda_{i}^{*}$, or complex conjugate pairs $\left(K_{j}=K_{i}^{*}\right)$, to ensure $\lambda_{j}=1 / \lambda_{i}^{*}$. The integration constants of the Riccati equations (66) have to be chosen such that $\alpha_{i}=1 / \alpha_{i}^{*}$ or $\alpha_{j}=1 / \alpha_{i}^{*}$, respectively.

For the particular seed solution (3) we obtain

$$
\alpha_{0}=-\frac{H^{*}}{H}
$$


Bäcklund transforms of the extreme Kerr near-horizon geometry

and

$$
\alpha_{i}=-\frac{\psi\left(\lambda_{i}, \theta\right)-c_{i} \psi\left(-\lambda_{i}, \theta\right)}{\chi\left(\lambda_{i}, \theta\right)+c_{i} \chi\left(-\lambda_{i}, \theta\right)} \quad(i=1,2, \ldots, 2 n)
$$

with

$$
\begin{aligned}
& \psi(\lambda, \theta)=\left[\chi\left(1 / \lambda^{*}, \theta\right)\right]^{*}=\frac{A\left(1+\lambda^{2}\right)+B\left(1-\lambda^{2}\right)+C \lambda}{\mathrm{e}^{-\mathrm{i} \theta}\left(\lambda-\mathrm{e}^{\mathrm{i} \theta}\right)^{2}}, \\
& A=\frac{\cos \theta+\mathrm{i}}{1+\mathrm{i}}, \quad B=\frac{(1-\mathrm{i}) \sin \theta}{\cos \theta-\mathrm{i}}
\end{aligned}
$$

and

$$
C=-(1+\mathrm{i})(\cos \theta-\mathrm{i}) .
$$

The constants $c_{i}$, which can also be chosen infinite [meaning $\alpha_{i}=\psi\left(-\lambda_{i}, \theta\right) / \chi\left(-\lambda_{i}, \theta\right)$, have to satisfy

$$
c_{i}=-c_{i}^{*} \quad\left(\text { for real } K_{i}\right) \quad \text { or } \quad c_{j}=-c_{i}^{*} \quad\left(\text { for pairs } K_{j}=K_{i}^{*}\right) .
$$

We mention that $\alpha_{0}$ solves the Riccati equation (6) with $\lambda_{i}$ replaced by 1 and can be expressed here as

$$
\alpha_{0}=-\lim _{\lambda \rightarrow 1} \frac{\psi(\lambda, \theta)+\mathrm{i} \psi(-\lambda, \theta)}{\chi(\lambda, \theta)-\mathrm{i} \chi(-\lambda, \theta)}
$$

(note that $\psi(1, \theta)=-\chi(1, \theta)=-\mathrm{i}, \psi(-1, \theta)=\chi(-1, \theta)=1$ ).

\section{Solutions with extreme Kerr near-horizon asymptotics}

For the discussion of the asymptotic behaviour as $r \rightarrow \infty$ a reformulation of (44) in terms of $n \times n$ determinants following [20] is useful, see also [21]. With

$$
r_{i} \equiv-\lambda_{i}\left(K_{i}-r \mathrm{e}^{-\mathrm{i} \theta}\right)=r \sqrt{\left(1-\frac{K_{i} \mathrm{e}^{\mathrm{i} \theta}}{r}\right)\left(1-\frac{K_{i} \mathrm{e}^{-\mathrm{i} \theta}}{r}\right)}
$$

one obtains

$$
\mathcal{E}=\mathcal{E}_{0} \frac{\operatorname{det}\left(\frac{\alpha_{p} r_{p}-\alpha_{q} r_{q}}{K_{p}-K_{q}}+\alpha_{0}\right)}{\operatorname{det}\left(\frac{\alpha_{p} r_{p}-\alpha_{q} r_{q}}{K_{p}-K_{q}}+1\right)}
$$

with

$$
p=1,3,5, \ldots, 2 n-1 ; \quad q=2,4,6, \ldots, 2 n .
$$

(This means: first row $p=1$, second row $p=3, \ldots, n$-th row $p=2 n-1$ and first column $q=2$, second column $q=4, \ldots, n$-th column $q=2 n$.) For $n=1$, Eq. (16) reduces to

$$
\mathcal{E}=\mathcal{E}_{0} \frac{\alpha_{1} r_{1}-\alpha_{2} r_{2}+\alpha_{0}\left(K_{1}-K_{2}\right)}{\alpha_{1} r_{1}-\alpha_{2} r_{2}+K_{1}-K_{2}}
$$


By means of an expansion in powers of $r^{-1}$ it can easily be verified from (5) and (91)-(12) that

$$
\alpha_{i}=-\frac{\psi\left(\lambda_{i}, \theta\right)}{\chi\left(\lambda_{i}, \theta\right)}+\mathcal{O}\left(r^{-2}\right)=F(\theta)+\mathcal{O}\left(r^{-1}\right) \quad \text { for } c_{i} \neq \infty
$$

and

$$
\alpha_{i}=\frac{\psi\left(-\lambda_{i}, \theta\right)}{\chi\left(-\lambda_{i}, \theta\right)}=G(\theta)+\mathcal{O}\left(r^{-1}\right) \quad \text { for } c_{i}=\infty
$$

with

$$
F(\theta)=\frac{i(\cos \theta+i)^{2}}{(\cos \theta-i)^{2}}
$$

and

$$
G(\theta)=\frac{i(\cos \theta+i)(6 i-15 \cos \theta-6 i \cos 2 \theta-\cos 3 \theta)}{(\cos \theta-i)(6 i+15 \cos \theta-6 i \cos 2 \theta+\cos 3 \theta)}
$$

Because of

$$
\lim _{r \rightarrow \infty} \frac{r_{i}}{r}=1
$$

see (15), we find (with $\mathcal{E}_{0}=\mathcal{E}_{\mathrm{NHG}}$ )

$$
\lim _{r \rightarrow \infty} \frac{\mathcal{E}}{\mathcal{E}_{\mathrm{NHG}}}=1
$$

for all $\theta$ with $F(\theta) \neq G(\theta)$ if $n$ of the $2 n$ constants $c_{i}$, say $c_{p}$ (with $p=1,3,5, \ldots, 2 n-1$ ), are chosen finite and the other ones, say $c_{q}$ (with $q=2,4,6, \ldots, 2 n$ ), are chosen infinite. This reduces the number of free real constants contained in the $K_{i}$ 's and $c_{i}$ 's from $4 n$ to $3 n$. For $n=1, c_{1} \neq \infty$ and $c_{2}=\infty$ means that $K_{1}$ and $K_{2}$ must be real, see (13). For $n>1$, pairs of complex conjugate $K_{i}$ 's are possible as well. It turns out that the so far excluded special values of $\theta$ defined by $F(\theta)=G(\theta)$ are the same values for which $\alpha_{0} \equiv-H^{*} / H=1$ holds $\left(\cos ^{2} \theta=2 \sqrt{3}-3\right)$, leading obviously to $\mathcal{E}=\mathcal{E}_{\mathrm{NHG}}$ for all $r$. Hence our solutions have the extreme Kerr near-horizon asymptotics whenever precisely $n$ of the $2 n$ constants $c_{i}$ are chosen infinites.

Explicit expressions for all metric functions can be calculated using the general Bäcklund formalism, see [22]. For convenience of the reader, the metric functions in the case $n=1$ are given in the Appendix.

\section{Conclusion}

Eqs (4, 5) with $\mathcal{E}_{0}=\mathcal{E}_{\mathrm{NHG}}$ and (8)-(12) represent a new hierarchy of solutions to the vacuum Einstein equations, the ( $2 n$-fold) Bäcklund transforms of the extreme Kerr nearhorizon geometry $(n=1,2,3, \ldots)$. For given $n$, the solution contains $4 n$ arbitrary real parameters. From a mathematical point of view, this result is an example of applying

$\S$ Note that these infinite constants can be identified with $c_{q}(q=2,4,6, \ldots, 2 n)$ without loss of generality since the expression (4) is invariant under simultaneous permutations of the $2 n$ indices of the $\lambda_{i}$ and $\alpha_{i}$. 
Bäcklund transformations to non-static and non-asymptotically flat seed solutions. As discussed in the Introduction, members of the $3 n$-parameter subfamily with extreme Kerr near-horizon asymptotics might be of particular interest. Of course, further investigations are needed to explore the physical significance of these solutions.

\section{Appendix}

For the case $n=1$, the three-parameter $\|$ family of solutions with extreme Kerr nearhorizon asymptotics leads to the following expressions for the metric functions in (2):

$$
\begin{aligned}
& f=\Re\left(\mathcal{E}_{\mathrm{NHG}} \frac{\alpha_{1} r_{1}-\alpha_{2} r_{2}+\alpha_{0}\left(K_{1}-K_{2}\right)}{\alpha_{1} r_{1}-\alpha_{2} r_{2}+K_{1}-K_{2}}\right), \\
& h=h_{0} \Omega^{4} K_{1}^{4} \psi_{1} \psi_{2} \psi_{1}^{*} \psi_{2}^{*}\left(\lambda_{1} \lambda_{2}^{*}+\lambda_{1}^{*} \lambda_{2}-\alpha_{1} \alpha_{2}^{*}-\alpha_{1}^{*} \alpha_{2}\right) f_{0}^{-2}
\end{aligned}
$$

and

$$
a=\left(a_{0} f_{0}-2 \Im Q\right) f^{-1}
$$

where

$$
\begin{aligned}
& h_{0}=h_{\mathrm{NHG}}=\frac{1}{4}\left(\cos ^{4} \theta+6 \cos ^{2} \theta-3\right), \\
& f_{0}=f_{\mathrm{NHG}}=\frac{4 \Omega^{2} r^{2} h_{0}}{\cos ^{2} \theta+1}, \\
& a_{0}=a_{\mathrm{NHG}}=-\frac{2 r \sin ^{2} \theta}{f_{0}\left(\cos ^{2} \theta+1\right)}, \\
& \psi_{1}=\psi\left(\lambda_{1}, \theta\right)-c_{1} \psi\left(-\lambda_{1}, \theta\right), \quad \psi_{2}=-\mathrm{i} \psi\left(-\lambda_{2}, \theta\right),
\end{aligned}
$$

and

$$
Q=\frac{\alpha_{1} r_{1}\left(K_{2}-r \cos \theta\right)-\alpha_{2} r_{2}\left(K_{1}-r \cos \theta\right)+\mathrm{i} a_{0} f_{0}\left(K_{1}-K_{2}\right)}{\alpha_{1} r_{1}-\alpha_{2} r_{2}+K_{1}-K_{2}}
$$

Note that

$$
\lim _{r \rightarrow \infty} \frac{f}{f_{0}}=\lim _{r \rightarrow \infty} \frac{h}{h_{0}}=\lim _{r \rightarrow \infty} \frac{a}{a_{0}}=1
$$

\section{References}

[1] Neugebauer G and Meinel R 1995 Phys. Rev. Lett. 753046

[2] Meinel R 1997 The rigidly rotating disk of dust and its black hole limit Proceedings of the 2nd Mexican School on Gravitation and Mathematical Physics arXiv:gr-qc/9703077

[3] Meinel R, Ansorg M, Kleinwächter A, Neugebauer G and Petroff D 2008 Relativistic Figures of Equilibrium (Cambridge: Cambridge University Press)

[4] Ansorg M, Kleinwächter A and Meinel R 2003 Astrophys. J. 582 L87

[5] Meinel R 2006 Class. Quantum Grav. 231359

[6] Bardeen J M and Horowitz G T 1999 Phys. Rev. D 60104030

$\|$ The three parameters are given by the two real constants $K_{1}, K_{2}$ and the imaginary constant $c_{1}$ (remember that $c_{2}=\infty$ ). 
[7] Novikov S, Manakov S V, Pitaevskii L P and Zakharov V E 1984 Theory of Solitons: The Inverse Scattering method (New York: Consultants Bureau)

[8] Belinski V and Verdaguer E 2001 Gravitational Solitons (Cambridge: Cambridge University Press)

[9] Rogers C and Schief W K 2002 Bäcklund and Darboux Transformations (Cambridge: Cambridge University Press)

[10] Harrison B K 1978 Phys. Rev. Lett. 411197

[11] Neugebauer G 1979 J. Phys. A: Math. Gen. 12 L67

[12] Amsel A J, Horowitz G T, Marolf D and Roberts M M 2009 JHEP09 044

[13] Dias O J C, Reall H S and Santos J E 2009 JHEP08 101

[14] Compère G 2017 Living Rev. Relativ. 201

[15] Ernst F J 1968 Phys. Rev. 1671175

[16] Kramer D and Neugebauer G 1968 Commun. Math. Phys. 10132

[17] Ernst F J 1977 J. Math. Phys. 18233

[18] Neugebauer G 1980 J. Phys. A: Math. Gen. 13 L19

[19] Neugebauer G 1980 J. Phys. A: Math. Gen. 131737

[20] Yamazaki M 1983 Phys. Rev. Lett. 501027

[21] Meinel R, Neugebauer G and Steudel H 1991 Solitonen: Nichtlineare Strukturen (Berlin: Akademie Verlag)

[22] Kramer D 1980 On the algebraic calculation of new solutions 9th International Conference on General Relativity and Gravitation in Jena, Germany (GR9) [Abstracts of Contributed Papers for the Discussion Groups, Volume 1, pp. 42-43] 